\title{
Unified treatment of nonrelativistic and quasirelativistic atomic integrals over complete orthonormal sets of $\Psi^{\alpha}$-exponential type orbitals
}

\author{
I. I. Guseinov
}

Received: 30 January 2008 / Accepted: 5 March 2008 / Published online: 2 August 2008

(C) The Author(s) 2008

\begin{abstract}
The new one- and two-electron nonrelativistic and quasirelativistic basic functions are introduced. The general analytical relations in terms of basic functions suggested are derived for the non- and quasi-relativistic atomic integrals over complete orthonormal sets of $\Psi^{\alpha}$-exponential type orbitals introduced by the author, where $\alpha=1,0,-1,-2, \ldots$ The relationships obtained are valid for the arbitrary values of quantum numbers and screening constants of orbitals.
\end{abstract}

Keywords $\Psi^{\alpha}$-Exponential type orbitals · Potential functions ·

Nonrelativistic atomic integrals · Quasirelativistic atomic integrals

\section{Introduction}

Relativistic calculations play a more and more important role in atomic, molecular, and nuclear physics [1,2]. Therefore, it is important to use appropriate and elegant sets of basis functions with well understood properties. In Ref. [3], we have presented the new sets of complete orthonormal sets of $\Psi^{\alpha}$-exponential type orbitals ( $\Psi^{\alpha}$-ETO) which might be useful for future applications based upon the use of $\Psi^{\alpha}$-ETO as basis functions in the nonrelativistic and relativistic LCAO theory, where $\alpha=1,0,-1,-2, \ldots$ Therefore, a large number of different sets of Hartree-Fock-Roothaan equations can be obtained with the help of single analytical relation of $\Psi^{\alpha}$-ETO which has the further advantage that the basic $\Psi^{\alpha}$-ETO, which are required, can be chosen properly according to the nature of the problems under consideration. This is rather important because the choice of the basis set will determine the rate of convergence of the resulting LCAO expansions. Therefore, the elaboration of algorithms for the evaluation

\footnotetext{
I. I. Guseinov $(\varangle)$

Department of Physics, Faculty of Arts and Sciences, Onsekiz Mart University, Canakkale, Turkey

e-mail: isguseinov@yahoo.com
} 
of matrix elements in the nonrelativistic and relativistic LCAO theory with $\Psi^{\alpha}$-ETO necessitates progress in the development of the methods to calculate the atomic and molecular integrals over $\Psi^{\alpha}$-ETO. The purpose of the present paper is to derive the new combined simple formulas for atomic integrals over complete orthonormal sets of $\Psi^{\alpha}$-ETO arising in nonrelativistic and relativistic theory of atoms.

\section{Expressions for non- and quasi-relativistic atomic integrals in terms of basic integrals}

The non- and quasi-relativistic (NQR) atomic integrals over complete orthonormal sets of $\Psi^{\alpha}$-ETO examined in the present work have the following form:

One-electron NQR integrals (or NQR potential functions)

$$
\begin{aligned}
& U_{p_{1} p_{1}^{\prime}}^{\alpha}\left(\zeta_{1} \zeta_{1}^{\prime}, \vec{r}_{o 2}\right)=\int \Psi_{p_{1}}^{\alpha^{*}}\left(\zeta_{1}, \vec{r}_{o 1}\right) \Psi_{p_{1}^{\prime}}^{\alpha}\left(\zeta_{1}^{\prime}, \vec{r}_{o 1}\right) O\left(r_{21}\right) d v_{1} \\
& U_{p_{1} p_{1}^{\prime}}^{\alpha i}\left(\zeta_{1} \zeta_{1}^{\prime}, \vec{r}_{o 2}\right)=\int \Psi_{p_{1}}^{\alpha^{*}}\left(\zeta_{1}, \vec{r}_{o 1}\right) \Psi_{p_{1}^{\prime}}^{\alpha}\left(\zeta_{1}^{\prime}, \vec{r}_{o 1}\right) O^{i}\left(\vec{r}_{21}\right) d v_{1} \\
& U_{p_{1} p_{1}^{\prime}}^{\alpha i j}\left(\zeta_{1} \zeta_{1}^{\prime}, \vec{r}_{o 2}\right)=\int \Psi_{p_{1}}^{\alpha^{*}}\left(\zeta_{1}, \vec{r}_{o 1}\right) \Psi_{p_{1}^{\prime}}^{\alpha}\left(\zeta_{1}^{\prime}, \vec{r}_{o 1}\right) O^{i j}\left(\vec{r}_{21}\right) d v_{1} \\
& V_{p_{1} p_{1}^{\prime}}^{\alpha i j}\left(\zeta_{1} \zeta_{1}^{\prime}, \vec{r}_{o 2}\right)=\int \Psi_{p_{1}}^{\alpha^{*}}\left(\zeta_{1}, \vec{r}_{o 1}\right) \Psi_{p_{1}^{\prime}}^{\alpha}\left(\zeta_{1}^{\prime}, \vec{r}_{o 1}\right) \mathcal{R}^{i j}\left(\vec{r}_{21}\right) d v_{1}
\end{aligned}
$$

Two-electron NQR integrals

$$
\begin{aligned}
& I_{p_{1} p_{1}^{\prime}, p_{2} p_{2}^{\prime}}^{\alpha}\left(\zeta_{1} \zeta_{1}^{\prime}, \zeta_{2} \zeta_{2}^{\prime}\right) \\
& \quad=\iint \Psi_{p_{1}}^{\alpha^{*}}\left(\zeta_{1}, \vec{r}_{o 1}\right) \Psi_{p_{1}^{\prime}}^{\alpha}\left(\zeta_{1}^{\prime}, \vec{r}_{o 1}\right) O\left(r_{21}\right) \Psi_{p_{2}}^{\alpha}\left(\zeta_{2}, \vec{r}_{o 2}\right) \Psi_{p_{2}^{\prime}}^{\alpha^{*}}\left(\zeta_{2}^{\prime}, \vec{r}_{o 2}\right) d v_{1} d v_{2} \\
& \quad=\int U_{p_{1} p_{1}^{\prime}}\left(\zeta_{1} \zeta_{1}^{\prime}, \vec{r}_{o 2}\right) \Psi_{p_{2}}^{\alpha}\left(\zeta_{2}, \vec{r}_{o 2}\right) \Psi_{p_{2}^{\prime}}^{\alpha^{*}}\left(\zeta_{2}^{\prime}, \vec{r}_{o 2}\right) d v_{2} \\
& I_{p_{1} p_{1}^{\prime}, p_{2} p_{2}^{\prime}}^{\alpha i}\left(\zeta_{1} \zeta_{1}^{\prime}, \zeta_{2} \zeta_{2}^{\prime}\right) \\
& \quad=\iint \Psi_{p_{1}}^{\alpha^{*}}\left(\zeta_{1}, \vec{r}_{o 1}\right) \Psi_{p_{1}^{\prime}}^{\alpha}\left(\zeta_{1}^{\prime}, \vec{r}_{o 1}\right) O^{i}\left(\vec{r}_{21}\right) \Psi_{p_{2}}^{\alpha}\left(\zeta_{2}, \vec{r}_{o 2}\right) \Psi_{p_{2}^{\prime}}^{\alpha^{*}}\left(\zeta_{2}^{\prime}, \vec{r}_{o 2}\right) d v_{1} d v_{2} \\
& \quad=\int U_{p_{1} p_{1}^{\prime}}^{\alpha i}\left(\zeta_{1} \zeta_{1}^{\prime}, \vec{r}_{o 2}\right) \Psi_{p_{2}}^{\alpha}\left(\zeta_{2}, \vec{r}_{o 2}\right) \Psi_{p_{2}^{\prime}}^{\alpha^{*}}\left(\zeta_{2}^{\prime}, \vec{r}_{o 2}\right) d v_{2}
\end{aligned}
$$




$$
\begin{aligned}
& I_{p_{1} p_{1}^{\prime}, p_{2} p_{2}^{\prime}}^{\alpha i j}\left(\zeta_{1} \zeta_{1}^{\prime}, \zeta_{2} \zeta_{2}^{\prime}\right) \\
& =\iint \Psi_{p_{1}}^{\alpha^{*}}\left(\zeta_{1}, \vec{r}_{o 1}\right) \Psi_{p_{1}^{\prime}}^{\alpha}\left(\zeta_{1}^{\prime}, \vec{r}_{o 1}\right) O^{i j}\left(\vec{r}_{21}\right) \Psi_{p_{2}}^{\alpha}\left(\zeta_{2}, \vec{r}_{o 2}\right) \Psi_{p_{2}^{\prime}}^{\alpha^{*}}\left(\zeta_{2}^{\prime}, \vec{r}_{o 2}\right) d v_{1} d v_{2} \text { (7a) } \\
& =\int U_{p_{1} p_{1}^{\prime}}^{\alpha i j}\left(\zeta_{1} \zeta_{1}^{\prime}, \vec{r}_{o 2}\right) \Psi_{p_{2}}^{\alpha}\left(\zeta_{2}, \vec{r}_{o 2}\right) \Psi_{p_{2}^{\prime}}^{\alpha^{*}}\left(\zeta_{2}^{\prime}, \vec{r}_{o 2}\right) d v_{2} \\
& \mathcal{L}_{p_{1} p_{1}^{\prime}, p_{2} p_{2}^{\prime}}^{\alpha i j}\left(\zeta_{1} \zeta_{1}^{\prime}, \zeta_{2} \zeta_{2}^{\prime}\right) \\
& =\int \Psi_{p_{1}}^{\alpha^{*}}\left(\zeta_{1}, \vec{r}_{o 1}\right) \Psi_{p_{1}^{\prime}}^{\alpha}\left(\zeta_{1}^{\prime}, \vec{r}_{o 1}\right) \mathcal{R}^{i j}\left(\vec{r}_{21}\right) \Psi_{p_{2}}^{\alpha}\left(\zeta_{2}, \vec{r}_{o 2}\right) \Psi_{p_{2}^{\prime}}^{\alpha^{*}}\left(\zeta_{2}^{\prime}, \vec{r}_{o 2}\right) d v_{1} d v_{2} \\
& =\int V_{p_{1} p_{1}^{\prime}}^{\alpha i j}\left(\zeta_{1} \zeta_{1}^{\prime}, \vec{r}_{o 2}\right) \Psi_{p_{2}}^{\alpha}\left(\zeta_{2}, \vec{r}_{o 2}\right) \Psi_{p_{2}^{\prime}}^{\alpha^{*}}\left(\zeta_{2}^{\prime}, \vec{r}_{o 2}\right) d v_{2} .
\end{aligned}
$$

Here $p_{k} \equiv n_{k} l_{k} m_{k}, p_{k}^{\prime} \equiv n_{k}^{\prime} l_{k}^{\prime} m_{k}^{\prime}, \quad \vec{r}_{21}=\vec{r}_{o 1}-\vec{r}_{o 2}, \vec{r}_{o 1}$ and $\vec{r}_{o 2}$ are the radius vectors of the electrons relative to a reference origin $0 ; i, j=0, \pm 1 ; x_{1}^{1}=x_{1}, x_{1}^{-1}=$ $y_{1}, x_{1}^{0}=z_{1}$ and $x_{2}^{1}=x_{2}, x_{2}^{-1}=y_{2}, x_{2}^{0}=z_{2}$ are the Cartesian coordinates of electrons; the normalized complex or real $\Psi^{\alpha}$-ETO are determined by [3]

$$
\begin{aligned}
& \Psi_{n l m}^{\alpha}(\zeta, \vec{r}) \\
& =(-1)^{\alpha}\left[\frac{(2 \zeta)^{3}(n-l-1) !}{(2 n)^{\alpha}[(n+l+1-\alpha) !]^{3}}\right]^{1 / 2}(2 \zeta r)^{l} e^{-\zeta r} L_{n+l+1-\alpha}^{2 l+2-\alpha}(2 \zeta r) S_{l m}(\theta, \varphi) .
\end{aligned}
$$

The operators $O\left(r_{21}\right), O^{i}\left(\vec{r}_{21}\right), O^{i j}\left(\vec{r}_{21}\right)$ and $\mathcal{R}^{i j}\left(\vec{r}_{21}\right)$ occurring in Eqs. $1-8$ are defined as [4]

$$
\begin{aligned}
O\left(r_{21}\right) & =\frac{1}{r_{21}} \\
O^{i}\left(\vec{r}_{21}\right) & =\frac{x_{21}^{i}}{r_{21}^{3}}=\frac{\partial}{\partial x_{2}^{i}}\left(\frac{1}{r_{21}}\right) \\
O^{i j}\left(\vec{r}_{21}\right) & =\frac{3 x_{21}^{i} x_{21}^{j}-\delta_{i j} r_{21}^{2}}{r_{21}^{5}}-\frac{4 \pi}{3} \delta_{i j} \delta\left(\vec{r}_{21}\right)=\frac{\partial^{2}}{\partial x_{2}^{i} \partial x_{2}^{j}}\left(\frac{1}{r_{21}}\right) \\
\mathcal{R}^{i j}\left(\vec{r}_{21}\right) & =\frac{x_{21}^{i} x_{21}^{j}}{r_{21}^{3}} .
\end{aligned}
$$

Here, the operators $O\left(r_{21}\right)$ and $\mathcal{R}^{i j}\left(\vec{r}_{21}\right)$ are the nonrelativistic Coulomb potential and the relativistic correction of Coulomb potential to the interactions between the electrons, respectively. The correction is due to the retardation of the electromagnetic field produced by an electron. The operators $O^{i}\left(\vec{r}_{21}\right)$ and $O^{i j}\left(\vec{r}_{21}\right)$ describe the spinorbit and spin-spin magnetic moment interactions of the two-electrons, respectively. 
We notice that the operators (10), (11) and (12) can also be used in study of the classical electrostatic potential, electric field and electric field gradient created by the electrons of an atom.

In order to evaluate the integrals (1)-(8), we use the following expansion relations for the electron charge densities of $\Psi^{\alpha}$-ETO established in previous paper [5]:

$$
\Psi_{p}^{\alpha}(\zeta, \vec{r}) \Psi_{p^{\prime}}^{\alpha^{*}}\left(\zeta^{\prime}, \vec{r}\right)=\frac{(2 z)^{3 / 2}}{\sqrt{4 \pi}} \sum_{\mu=1}^{n+n^{\prime}-1} \sum_{\nu=0}^{\mu-1} \sum_{\sigma=-v}^{v} B_{p p^{\prime}}^{\alpha q}(\eta) \Psi_{q}^{\alpha}(z, \vec{r})
$$

where $q \equiv \mu \nu \sigma, \eta=\zeta / \zeta^{\prime}$ and $z=\zeta+\zeta^{\prime}$. Taking into account Eq. 14 in 1-8 we obtain the expressions in terms of basic integrals: for one-electron NQR integrals

$$
\begin{aligned}
U_{p_{1} p_{1}^{\prime}}^{\alpha}\left(\zeta_{1} \zeta_{1}^{\prime}, \vec{r}_{o 2}\right) & =\left(2 z_{1}\right)^{3 / 2} \sum_{\nu_{1} \sigma_{1}} B_{p_{1} p_{1}^{\prime}}^{\alpha q_{1}}\left(\eta_{1}\right) J_{q_{1}}^{\alpha}\left(z_{1}, \vec{r}_{o 2}\right) \\
U_{p_{1} p_{1}^{\prime}}^{\alpha i}\left(\zeta_{1} \zeta_{1}^{\prime}, \vec{r}_{o 2}\right) & =\left(2 z_{1}\right)^{3 / 2} \sum_{\nu_{1} \sigma_{1}} B_{p_{1} p_{1}^{\prime}}^{\alpha q_{1}}\left(\eta_{1}\right) J_{q_{1}}^{\alpha i}\left(z_{1}, \vec{r}_{o 2}\right) \\
U_{p_{1} p_{1}^{\prime}}^{\alpha i j}\left(\zeta_{1} \zeta_{1}^{\prime}, \vec{r}_{o 2}\right) & =\left(2 z_{1}\right)^{3 / 2} \sum_{\nu_{1} \sigma_{1}} B_{p_{1} p_{1}^{\prime}}^{\alpha q_{1}}\left(\eta_{1}\right) J_{q_{1}}^{\alpha i j}\left(z_{1}, \vec{r}_{o 2}\right) \\
V_{p_{1} p_{1}^{\prime}}^{\alpha i j}\left(\zeta_{1} \zeta_{1}^{\prime}, \vec{r}_{o 2}\right) & =\left(2 z_{1}\right)^{3 / 2} \sum_{\nu_{1} \sigma_{1}} B_{p_{1} p_{1}^{\prime}}^{\alpha q_{1}}\left(\eta_{1}\right) \Lambda_{q_{1}}^{\alpha i j}\left(z_{1}, \vec{r}_{o 2}\right),
\end{aligned}
$$

for two-electron NQR integrals

$$
\begin{aligned}
& I_{p_{1} p_{1}^{\prime}, p_{2} p_{2}^{\prime}}^{\alpha}\left(\zeta_{1} \zeta_{1}^{\prime}, \zeta_{2} \zeta_{2}^{\prime}\right)=\left(4 z_{1} z_{2}\right)^{3 / 2} \sum_{\nu_{1} \sigma_{1}} \sum_{v_{2} \sigma_{2}} B_{p_{1} p_{1}^{\prime}}^{\alpha q_{1}}\left(\eta_{1}\right) B_{p_{2} p_{2}^{\prime}}^{\alpha q_{2}}\left(\eta_{2}\right) K_{q_{1} q_{2}}^{\alpha}\left(z_{1}, z_{2}\right) \\
& I_{p_{1} p_{1}^{\prime}, p_{2} p_{2}^{\prime}}^{\alpha i}\left(\zeta_{1} \zeta_{1}^{\prime}, \zeta_{2} \zeta_{2}^{\prime}\right)=\left(4 z_{1} z_{2}\right)^{3 / 2} \sum_{\nu_{1} \sigma_{1}} \sum_{\nu_{2} \sigma_{2}} B_{p_{1} p_{1}^{\prime}}^{\alpha q_{1}}\left(\eta_{1}\right) B_{p_{2} p_{2}^{\prime}}^{\alpha q_{2}}\left(\eta_{2}\right) K_{q_{1} q_{2}}^{\alpha i}\left(z_{1}, z_{2}\right) \\
& I_{p_{1} p_{1}^{\prime}, p_{2} p_{2}^{\prime}}^{\alpha i j}\left(\zeta_{1} \zeta_{1}^{\prime}, \zeta_{2} \zeta_{2}^{\prime}\right)=\left(4 z_{1} z_{2}\right)^{3 / 2} \sum_{\nu_{1} \sigma_{1}} \sum_{\nu_{2} \sigma_{2}} B_{p_{1} p_{1}^{\prime}}^{\alpha q_{1}}\left(\eta_{1}\right) B_{p_{2} p_{2}^{\prime}}^{\alpha q_{2}}\left(\eta_{2}\right) K_{q_{1} q_{2}}^{\alpha i j}\left(z_{1}, z_{2}\right) \\
& \mathcal{L}_{p_{1} p_{1}^{\prime}, p_{2} p_{2}^{\prime}}^{\alpha i j}\left(\zeta_{1} \zeta_{1}^{\prime}, \zeta_{2} \zeta_{2}^{\prime}\right)=\left(4 z_{1} z_{2}\right)^{3 / 2} \sum_{\nu_{1} \sigma_{1}} \sum_{\nu_{2} \sigma_{2}} B_{p_{1} p_{1}^{\prime}}^{\alpha q_{1}}\left(\eta_{1}\right) B_{p_{2} p_{2}^{\prime}}^{\alpha q_{2}}\left(\eta_{2}\right) L_{q_{1} q_{2}}^{\alpha i j}\left(z_{1}, z_{2}\right)
\end{aligned}
$$

The basic integrals occurring in these formulae are defined as for one-electron basic integrals 


$$
\begin{aligned}
J_{q_{1}}^{\alpha}\left(z_{1}, \vec{r}_{2}\right) & =\frac{1}{\sqrt{4 \pi}} \int \Psi_{q_{1}}^{\alpha *}\left(z_{1}, \vec{r}_{1}\right) O\left(r_{21}\right) d v_{1} \\
J_{q_{1}}^{\alpha i}\left(z_{1}, \vec{r}_{2}\right) & =\frac{1}{\sqrt{4 \pi}} \int \Psi_{q_{1}}^{\alpha *}\left(z_{1}, \vec{r}_{1}\right) O^{i}\left(\vec{r}_{21}\right) d v_{1} \\
J_{q_{1}}^{\alpha i j}\left(z_{1}, \vec{r}_{2}\right) & =\frac{1}{\sqrt{4 \pi}} \int \Psi_{q_{1}}^{\alpha *}\left(z_{1}, \vec{r}_{1}\right) O^{i j}\left(\vec{r}_{21}\right) d v_{1} \\
\Lambda_{q_{1}}^{\alpha i j}\left(z_{1}, \vec{r}_{2}\right) & =\frac{1}{\sqrt{4 \pi}} \int \Psi_{q_{1}}^{\alpha *}\left(z_{1}, \vec{r}_{1}\right) \mathcal{R}^{i j}\left(\vec{r}_{21}\right) d v_{1},
\end{aligned}
$$

for two-electron basic integrals

$$
\begin{aligned}
& K_{q_{1} q_{2}}^{\alpha}\left(z_{1}, z_{2}\right)=\frac{1}{\sqrt{4 \pi}} \int J_{q_{1}}^{\alpha}\left(z_{1}, \vec{r}_{2}\right) \Psi_{q_{2}}^{\alpha}\left(z_{2}, \vec{r}_{2}\right) d v_{2} \\
& K_{q_{1} q_{2}}^{\alpha i}\left(z_{1}, z_{2}\right)=\frac{1}{\sqrt{4 \pi}} \int J_{q_{1}}^{\alpha i}\left(z_{1}, \vec{r}_{2}\right) \Psi_{q_{2}}^{\alpha}\left(z_{2}, \vec{r}_{2}\right) d v_{2} \\
& K_{q_{1} q_{2}}^{\alpha i j}\left(z_{1}, z_{2}\right)=\frac{1}{\sqrt{4 \pi}} \int J_{q_{1}}^{\alpha i j}\left(z_{1}, \vec{r}_{2}\right) \Psi_{q_{2}}^{\alpha}\left(z_{2}, \vec{r}_{2}\right) d v_{2} \\
& L_{q_{1} q_{2}}^{\alpha i j}\left(z_{1}, z_{2}\right)=\frac{1}{\sqrt{4 \pi}} \int \Lambda_{q_{1}}^{\alpha i j}\left(z_{1}, \vec{r}_{2}\right) \Psi_{q_{2}}^{\alpha}\left(z_{2}, \vec{r}_{2}\right) d v_{2},
\end{aligned}
$$

where $\vec{r}_{1} \equiv \vec{r}_{o 1}$ and $\vec{r}_{2} \equiv \vec{r}_{o 2}$.

Thus, the one- and two-electron NQR atomic integrals are expressed through the basic integrals defined by Eqs. 23-30.

\section{NQR basic functions}

In order to evaluate the basic integrals and, therefore, the NQR atomic integrals we introduce the following basic functions:

One-electron basic functions

$$
f_{\mu \nu, \kappa m}^{\alpha t k}(z, \vec{r})=f_{\mu \nu}^{\alpha t k}(z, r)\left(\frac{4 \pi}{2 \kappa+1}\right)^{1 / 2} S_{\kappa m}^{*}(\theta, \varphi),
$$

two-electron basic functions

$$
\mathrm{D}_{\mu_{1} v_{1}, \kappa m ; \mu_{2} v_{2} \sigma_{2}}^{\alpha t k}\left(z_{1}, z_{2}\right)=\frac{1}{\sqrt{4 \pi}} \int f_{\mu_{1} v_{1}, \kappa m}^{\alpha t k}\left(z_{1}, \vec{r}_{2}\right) \Psi_{\mu_{2} v_{2} \sigma_{2}}^{\alpha}\left(z_{2}, \vec{r}_{2}\right) d v_{2}
$$

Using method set out in Ref. [3], it is easy to express Eqs. 31 and 32 through the basic potential functions $f_{\mu \nu, \kappa m}^{t k}(z, \vec{r})$ (see Ref. [6]):

$$
f_{\mu \nu, \kappa m}^{\alpha t k}(z, \vec{r})=\sum_{\mu^{\prime}=v+1}^{\mu} \omega_{\mu \mu^{\prime}}^{\alpha \nu} f_{\mu^{\prime} v, \kappa m}^{t k}(z, \vec{r}),
$$


where

$$
\begin{aligned}
D_{\mu_{1} \nu_{1}, \kappa m ; \mu_{2} \nu_{2} \sigma_{2}}^{\alpha t k}\left(z_{1}, z_{2}\right) & =\sum_{\mu_{1}^{\prime}=v_{1}+1}^{\mu_{1}} \sum_{\mu_{2}^{\prime}=v_{2}+1}^{\mu_{2}} \omega_{\mu_{1} \mu_{1}^{\prime}}^{\alpha \nu_{1}} \omega_{\mu_{2} \mu_{2}^{\prime}}^{\alpha \nu_{2}} D_{\mu_{1}^{\prime} \nu_{1}, \kappa m ; \mu_{2}^{\prime} \nu_{2} \sigma_{2}}^{t k}\left(z_{1}, z_{2}\right) \\
f_{\mu \nu, \kappa m}^{t k}(z, \vec{r}) & =f_{\mu \nu}^{t k}(z, r)\left(\frac{4 \pi}{2 \kappa+1}\right)^{1 / 2} S_{\kappa m}^{*}(\theta, \varphi) \\
D_{\mu_{1} v_{1}, \kappa m ; \mu_{2} v_{2} \sigma_{2}}^{t k}\left(z_{1}, z_{2}\right) & =\frac{1}{\sqrt{4 \pi}} \int f_{\mu_{1} \nu_{1}, \kappa m}^{t k}\left(z_{1}, \vec{r}_{2}\right) \chi_{\mu_{2} \nu_{2} \sigma_{2}}\left(z_{2}, \vec{r}_{2}\right) d v_{2}
\end{aligned}
$$

See Ref. [3] for the exact definition of coefficients $\omega_{\mu \mu^{\prime}}^{\alpha \nu}$. Here, $\chi_{\mu_{2} v_{2} \sigma_{2}}\left(z_{2}, \vec{r}_{2}\right)$ are the normalized Slater type orbitals and

$$
\begin{aligned}
f_{\mu \nu, \kappa m}^{t k}(z, \vec{r})= & \frac{N_{\mu \nu}^{t}(2 z)}{(\mu+v+1) !(z r)^{v+t+1}} \\
& \times\left(\gamma(\mu+v+2, z r)+\delta_{k 0}(z r)^{2 v+1} \Gamma(\mu-v+1, z r)\right. \\
& \left.-\delta_{k 2} \frac{(z r)^{\mu+v+2}}{2 v+3} e^{-z r}\right)\left(\frac{4 \pi}{2 \kappa+1}\right)^{1 / 2} S_{\kappa m}^{*}(\theta, \varphi) \\
D_{\mu_{1} v_{1}, \kappa m ; \mu_{2} \nu_{2} \sigma_{2}}^{t k}\left(z_{1}, z_{2}\right)= & \delta_{\kappa \nu_{2}} \delta_{m \sigma_{2}} A_{\mu_{1} \nu_{1}, \kappa, \mu_{2}}^{t}\left(z_{1}, z_{2}\right) \\
& \times\left(\frac{F\left(1, \mu_{1}+\mu_{2}+3 ; \mu_{1}+v_{1}+3 ; 1 /(1+\eta)\right)}{\mu_{1}+v_{1}+2}\right) \\
& \left.+\frac{F\left(1, \mu_{1}+\mu_{2}+3 ; \mu_{2}+v_{1}+3 ; \eta /(1+\eta)\right)}{\mu_{2}+v_{1}+2}\right)
\end{aligned}
$$

where $0 \leq t \leq 2,0 \leq k \leq t$ and

$$
\begin{aligned}
N_{\mu \nu}^{t}(2 z) & =2^{\mu-t+1}(\mu+v+1) !\left[\frac{(2 z)^{2 t-1}}{(2 v+1)(2 \mu) !}\right]^{1 / 2} \\
A_{\mu_{1} \nu_{1}, \kappa, \mu_{2}}^{t}\left(z_{1}, z_{2}\right) & =\frac{2^{\mu_{1}+\mu_{2}+1}\left(\mu_{1}+\mu_{2}+2\right) ! \eta^{\mu_{2}+1 / 2}}{\left[\left(2 \nu_{1}+1\right)(2 \kappa+1)\left(2 \mu_{1}\right) !\left(2 \mu_{2}\right) !\right]^{\frac{1}{2}} z_{1}^{2}(1+\eta)^{\mu_{1}+\mu_{2}+3}} .
\end{aligned}
$$

Here $\Gamma(\alpha), \gamma(\alpha, x), \Gamma(\alpha, x)$ and $F(1, \mu ; v ; x)$ are the gamma and hypergeometric functions, respectively [7].

With the derivation of Eqs. 37 and 38 we used the properties of gamma and hypergeometric functions and the following relations [7];

$$
\begin{aligned}
\Gamma(\alpha) & =\gamma(\alpha, x)+\Gamma(\alpha, x) \\
\int_{0}^{\infty} x^{\mu-1} e^{-\beta x} \gamma(\nu, \alpha x) d x & =\frac{\alpha^{v} \Gamma(\mu+v)}{v(\alpha+\beta)^{\mu+v}} F\left(1, \mu+v ; v+1 ; \frac{\alpha}{\alpha+\beta}\right) \\
\int_{0}^{\infty} x^{\mu-1} e^{-\beta x} \Gamma(\nu, \alpha x) d x & =\frac{\alpha^{v} \Gamma(\mu+v)}{\mu(\alpha+\beta)^{\mu+v}} F\left(1, \mu+v ; \mu+1 ; \frac{\beta}{\alpha+\beta}\right)
\end{aligned}
$$


For the determination of values of one-electron basic functions at the origin we perform the limit $\vec{r} \rightarrow 0$ in Eq. 37. Then, we obtain:

$$
f_{\mu \nu, \kappa m}^{\alpha t k}(z, 0)=\delta_{m 0} \sum_{\mu^{\prime}=v+1}^{\mu} \omega_{\mu \mu^{\prime}}^{\alpha \nu} f_{\mu^{\prime} v}^{t k}(z, 0),
$$

where

$$
f_{\mu \nu}^{t k}(z, 0)=\delta_{k 0} N_{\mu \nu}^{t}(2 z)\left\{\begin{array}{ll}
\frac{(\mu-v) !}{(\mu+v+1) !(t-v) !} & \text { for } t \geq v \\
0 & \text { for } t<v
\end{array}\right. \text {. }
$$

\section{Expressions for basic integrals in terms of basic functions}

In order to derive the formulae for basic integrals through the basic functions we take into account Eqs. 33 and 34 in 23-30. Then, we find:

$$
\begin{aligned}
& J_{q}^{\alpha}\left(z, \vec{r}_{2}\right)=f_{\mu \nu, v \sigma}^{\alpha 00}\left(z, \vec{r}_{2}\right) \\
& J_{q}^{\alpha i}\left(z, \vec{r}_{2}\right)=\sum_{m=-(\nu-1)}^{\nu-1} a_{\nu \sigma, m}^{i} f_{\mu \nu, v-1 m}^{\alpha 10}\left(z, \vec{r}_{2}\right)-(2 v+1)\left(\frac{x_{2}^{i}}{r_{2}}\right) f_{\mu \nu, v \sigma}^{\alpha 11}\left(z, \vec{r}_{2}\right) \\
& J_{q}^{\alpha i j}\left(z, \vec{r}_{2}\right)=\sum_{m=-(v-2)}^{\nu-2} a_{\nu \sigma, m}^{i j} f_{\mu \nu, \nu-2 m}^{\alpha 20}\left(z, \vec{r}_{2}\right)-(2 v+1) \\
& \times \sum_{m=-(\nu-1)}^{\nu-1}\left[a_{\nu \sigma, m}^{i}\left(\frac{x_{2}^{j}}{r_{2}}\right)+a_{\nu \sigma, m}^{j}\left(\frac{x_{2}^{i}}{r_{2}}\right)\right] f_{\mu \nu, v-1 m}^{\alpha 21}\left(z, \vec{r}_{2}\right) \\
& -(2 v+1) \delta_{i j} f_{\mu \nu, v \sigma}^{\alpha 21}\left(z, \vec{r}_{2}\right) \\
& +(2 v+1)(2 v+3)\left(\frac{x_{2}^{i}}{r_{2}}\right)\left(\frac{x_{2}^{j}}{r_{2}}\right) f_{\mu \nu, v \sigma}^{\alpha 22}\left(z, \vec{r}_{2}\right)-\frac{\sqrt{4 \pi}}{3} \delta_{i j} \Psi_{\mu \nu \sigma}^{\alpha *}\left(z, \vec{r}_{2}\right) \\
& \Lambda_{q}^{\alpha i j}\left(z, \vec{r}_{2}\right)=\frac{1}{3} \sum_{l m, L M}(2 L+1)(2 l+1)^{1 / 2} C^{l|m|}(1 j, v \sigma) A_{j \sigma}^{m} \\
& \times C^{L|M|}(1 i, \operatorname{lm}) A_{i m}^{M} f_{\mu L, L M}^{\alpha 00}\left(z, \vec{r}_{2}\right),
\end{aligned}
$$

for two-electron basic integrals

$$
\begin{aligned}
K_{q_{1} q_{2}}^{\alpha}\left(z_{1}, z_{2}\right)= & D_{\mu_{1} v_{1}, v_{1} \sigma_{1} ; \mu_{2} v_{2} \sigma_{2}}^{\alpha 00}\left(z_{1}, z_{2}\right) \\
K_{q_{1} q_{2}}^{\alpha i}\left(z_{1}, z_{2}\right)= & \sum_{m_{1}=-\left(v_{1}-1\right)}^{\nu_{1}-1} a_{\nu_{1} \sigma_{1}, m_{1}}^{i} D_{\mu_{1} v_{1}, v_{1}-1 m_{1} ; \mu_{2} v_{2} \sigma_{2}}^{\alpha 10}\left(z_{1}, z_{2}\right) \\
& -\left(2 v_{1}+1\right) D_{\mu_{1} \nu_{1}, v_{1} \sigma_{1} ; \mu_{2} v_{2} \sigma_{2}}^{\alpha 11, i}\left(z_{1}, z_{2}\right)
\end{aligned}
$$




$$
\begin{aligned}
& K_{q_{1} q_{2}}^{\alpha i j}\left(z_{1}, z_{2}\right)=\sum_{m_{1}=-\left(v_{1}-2\right)}^{\nu_{1}-2} a_{v_{1} \sigma_{1}, m_{1}}^{i j} D_{\mu_{1} v_{1}, \nu_{1}-2 m_{1} ; \mu_{2} \nu_{2} \sigma_{2}}^{\alpha 20}\left(z_{1}, z_{2}\right) \\
& -\left(2 v_{1}+1\right) \sum_{m_{1}=-\left(v_{1}-1\right)}^{v_{1}-1}\left[a_{v_{1} \sigma_{1}, m_{1}}^{i} D_{\mu_{1} v_{1}, \nu_{1}-1 m_{1} ; \mu_{2} v_{2} \sigma_{2}}^{\alpha 21, j}\left(z_{1}, z_{2}\right)\right. \\
& \left.+a_{\nu_{1} \sigma_{1}, m_{1}}^{j} D_{\mu_{1} v_{1}, v_{1}-1 m_{1} ; \mu_{2} v_{2} \sigma_{2}}^{\alpha 21, i}\left(z_{1}, z_{2}\right)\right] \\
& -\left(2 v_{1}+1\right) \delta_{i j} D_{\mu_{1} v_{1}, v_{1} \sigma_{1} ; \mu_{2} v_{2} \sigma_{2}}^{\alpha 21}\left(z_{1}, z_{2}\right)+\left(2 v_{1}+1\right)\left(2 v_{1}+3\right) \\
& \times D_{\mu_{1} v_{1}, v_{1} \sigma_{1} ; \mu_{2} v_{2} \sigma_{2}}^{\alpha 22, i}\left(z_{1}, z_{2}\right) \\
& -\frac{1}{3} \delta_{i j} S_{\mu_{1} v_{1} \sigma_{1}, \mu_{2} v_{2} \sigma_{2}}^{\alpha}\left(z_{1}, z_{2}\right) \\
& L_{q_{1} q_{2}}^{\alpha i j}\left(z_{1}, z_{2}\right)=\frac{1}{3} \sum_{l m, L M}[(2 L+1)(2 l+1)]^{1 / 2} C^{l|m|}\left(1 j, v_{1} \sigma_{1}\right) A_{j \sigma_{1}}^{m} \\
& \times C^{L|M|}(1 i, \operatorname{lm}) A_{i m}^{M} D_{\mu_{1} L, L M ; \mu_{2} v_{2} \sigma_{2}}^{\alpha 00}\left(z_{1}, z_{2}\right),
\end{aligned}
$$

where

$$
S_{\mu_{1} v_{1} \sigma_{1}, \mu_{2} v_{2} \sigma_{2}}^{\alpha}\left(z_{1}, z_{2}\right)=\int \Psi_{\mu_{1} v_{1} \sigma_{1}}^{\alpha *}\left(z_{1}, \vec{r}_{2}\right) \Psi_{\mu_{2} v_{2} \sigma_{2}}^{\alpha}\left(z_{2}, \vec{r}_{2}\right) d v_{2}
$$

are the atomic overlap integrals and

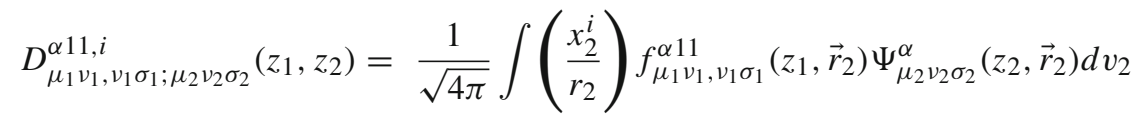

$$
\begin{aligned}
& =\sum_{l m} \frac{2 l+1}{\left[3\left(2 v_{1}+1\right)\right]^{1 / 2}} C^{l|m|}\left(1 i, v_{1} \sigma_{1}\right) A_{i \sigma_{1}}^{m} \\
& \times D_{\mu_{1} v_{1}, l m ; \mu_{2} v_{2} \sigma_{2}}^{\alpha 11}\left(z_{1}, z_{2}\right) \\
& D_{\mu_{1} v_{1}, v_{1}-1 m_{1} ; \mu_{2} v_{2} \sigma_{2}}^{\alpha 21, i}\left(z_{1}, z_{2}\right)=\frac{1}{\sqrt{4 \pi}} \int\left(\frac{x_{2}^{i}}{r_{2}}\right) f_{\mu_{1} \nu_{1}, v_{1}-1 m_{1}}^{\alpha 21}\left(z_{1}, \vec{r}_{2}\right) \Psi_{\mu_{2} v_{2} \sigma_{2}}^{\alpha}\left(z_{2}, \vec{r}_{2}\right) d v_{2} \\
& =\sum_{l m} \frac{2 l+1}{\left[3\left(2 v_{1}-1\right)\right]^{1 / 2}} C^{l|m|}\left(1 i, v_{1}-1 m_{1}\right) A_{i m_{1}}^{m} \\
& \times D_{\mu_{1} v_{1}, l m ; \mu_{2} v_{2} \sigma_{2}}^{\alpha 21}\left(z_{1}, z_{2}\right)
\end{aligned}
$$




$$
\begin{aligned}
D_{\mu_{1} v_{1}, \nu_{1} \sigma_{1} ; \mu_{2} v_{2} \sigma_{2}}^{\alpha 22, i}\left(z_{1}, z_{2}\right)= & \frac{1}{\sqrt{4 \pi}} \int\left(\frac{x_{2}^{i}}{r_{2}}\right)\left(\frac{x_{2}^{j}}{r_{2}}\right) \\
& \times f_{\mu_{1} v_{1}, v_{1} \sigma_{1}}^{\alpha 22}\left(z_{1}, \vec{r}_{2}\right) \Psi_{\mu_{2} v_{2} \sigma_{2}}^{\alpha}\left(z_{2}, \vec{r}_{2}\right) d v_{2} \\
= & \frac{1}{3} \sum_{l m, L M}(2 L+1)\left(\frac{2 l+1}{2 v_{1}+1}\right)^{1 / 2} C^{l|m|}\left(1 j, v_{1} \sigma_{1}\right) A_{j \sigma_{1}}^{m} \\
& \times C^{L|M|}(1 i, l m) A_{i m}^{M} D_{\mu_{1} v_{1}, L M ; \mu_{2} v_{2} \sigma_{2}}^{\alpha 22}\left(z_{1}, z_{2}\right) .
\end{aligned}
$$

Taking into account the formulae obtained for basic functions $f^{\alpha t k}$ and $D^{\alpha t k}$ all of the one- and two-electron nonrelativistic and quasirelativistic atomic integrals over $\Psi^{\alpha}$-ETO, Eqs. 1-8, can be calculated.

Open Access This article is distributed under the terms of the Creative Commons Attribution Noncommercial License which permits any noncommercial use, distribution, and reproduction in any medium, provided the original author(s) and source are credited.

\section{References}

1. P. Pyykko, Relativistic Theory of Atoms and Molecules I-III (Springer, Berlin, 1986, 1993 and 2000)

2. I.P. Grant, Relativistic Quantum Theory of Atoms and Molecules (Springer, 2006)

3. I.I. Guseinov, Int. J. Quantum Chem. 90, 114 (2002)

4. H.A. Bethe, E.E. Salpeter, Quantum Mechanics of One- and Two-Electron Atoms (Berlin, Springer, 1957)

5. I.I. Guseinov, J. Math. Chem. 42, 991 (2007)

6. I.I. Guseinov, J. Phys. A: Math. Gen. 37, 957 (2004)

7. I.S. Gradshteyn, I.M. Ryzhik, Tables of Integrals, Sums, Series and Products, 4th edn. (Academic, New York, 1980) 